Ann. Génét., Sél. anim., I973, 5 (4), 499-506.

\title{
CONSÉQUENCES
}

\section{DE L'HYPERTROPHIE MUSCULAIRE HÉRÉDITAIRE SUR LA TRAME CONJONCTIVE DU MUSCLE DE BOVIN}

\author{
B.-L. DUMONT et O. SCHMITT \\ Laboratoire de recherches sur la viande, \\ Centre national de Recherches zootechniques, I. N. R. A., \\ 78350 Jouy en Josas
}

Par rapport aux individus normaux de même race, de même sexe et de même poids de muscle, les sujets manifestant le caractère "culard " (hypertrophie musculaire héréditaire) présentent des différences sensibles dans l'importance relative des muscles (Dumont et Boccard, 1972).

Sur le plan de la composition chimique, il a été établi que les muscles des animaux " culards" ont des teneurs notablement plus faibles en tissu conjonctif que ceux des sujets normaux (LAWRIE et al., I964; BOCCARD et al., 1969) et il a été suggéré que ce caractère pourrait être l'une des conséquences de la perturbation du métabolisme du tissu conjonctif qui frappe ce type d'animal (BocCard, 1964). L'analyse histologique de la musculature a porté sur la dimension et sur le nombre des fibres musculaires (OUha youn et BEAUMONT, I968). En ce qui concerne les caractères du périmysium, les études ont été peu nombreuses et sont relativement discordantes. Selon Kidwell et al. (I952), l'examen microscopique du muscle d'un taurillon Africander $\times$ Angus de quatorze mois ne révélait pas de grosses " anomalies histologiques " (" gross histological abnormalities ") alors que BoccARD et al. (1969) rapportent l'existence de différences dans de nombreux aspects du périmysium, à partir d'examens portant sur des échantillons de muscles de bœufs de trois ans de race Charolaise de type normal et de type hypertrophié. Kidwell et al. (I952) signalent d'autre part, sans donner de détails, que le muscle du bovin hypertrophié présente macroscopiquement une texture plus grossière, avec des lames de tissu conjonctif longitudinal plus distinctes, et indiquent qu'il est généralement admis que la viande des animaux à forte musculature est plus grossière que la normale.

La présente note rapporte les résultats de la comparaison de la trame du tissu conjonctif intramusculaire de bovins de type normal et de type "culard". 


\section{I. - MATÉRIEL ET MÉTHODES}

L'étude a porté sur deux taurillons Charolais de quinze mois. Les muscles ont été choisis de façon à disposer d'une large gamme de degrés d'hypertrophie pondérale définie selon DumoNT et BoCCARD (1972) par le rapport :

\section{Poids du muscle " culard "- poids du muscle normal \\ Poids du muscle normal}

Ces muscles étaient les suivants :

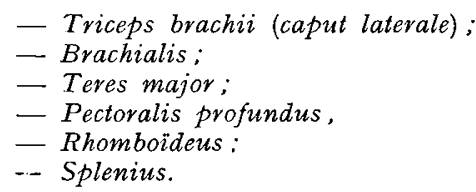

Pour chacun des muscles on a prélevé une tranche de trois centimètres d'épaisseur intéressant la surface totale dans sa partie la plus développée (correspondant à la portion médiane du muscle, sauf pour le muscle Triceps brachii (caput laterale). On a relevé ensuite le contour de l'échantillon, placé sur une feuille de papier, afin d'en mesurer la section. A partir des valeurs obtenues on a défini un degré d'hypertrophie morphologique par la valeur du rapport :

$\underline{\text { Surface du muscle " culard "— surface du muscle normal }}$

Après traitement histologique des tranches de muscle selon la méthode d'analyse précédemment décrite (Schmitt et Dumont, I969), des coupes, de I $5 \mu$ d'épaisseur, de la surface totale du muscle ont été colorées à la picro-fuschine de VAN Gieson. Les coupes homologues ont été comparées à différents grandissements compris entre $\mathrm{I}$ (ce qui correspond à un examen macroscopique normal) et I 5 .

\section{II. 一 RÉSULTATS}

Dans le tableau i sont décrits les animaux étudiés et les caractéristiques des muscles examinés. Les coupes sont présentées dans les figures i à 5 .

D'une manière générale, les sections transversales des muscles sont plus grandes chez le sujet " culard " que chez le normal. Cette hypertrophie morphologique s'observe même dans le cas où les rapports des masses des muscles homologues des deux types d'animaux sont très inférieurs à I. Cette discordance suggère l'existence, au sein de la musculature des bovins "culards ", d'une hypertrophie de la forme indépendante de l'hypertrophie en masse.

A faible grandissement, l'examen des coupes révèle une nette différence entre les muscles des deux types. L'impression générale laissée par l'observation des muscles de type hypertrophié est une nette réduction du tissu conjonctif normalement apparent à ce grandissement. Ce fait est illustré par les figures I à 4 , qui montrent, en plus, que le phénomène variable, d'un muscle à un autre, est particulièrement accentué dans le cas du muscle Triceps brachii (caput laterale), à la fois le plus hypertrophié en masse et en forme des muscles étudiés ici.

Sur le plan de l'organisation du muscle, ce caractère se traduit chez le " culard " par un amoindrissement de la trame générale du tissu conjonctif définissant les grandes unités d'organisation musculaire ou myoskhènes (Sснмiтt et Dumont, 1969).

Le nombre des myoskhènes est équivalent, pour chacun des muscles, dans les deux types d'animaux. Leurs dimensions sont accrues chez les muscles du "culard ", dans les mêmes proportions que les sections des muscles. 
TABLEAU I

\begin{tabular}{|c|c|c|c|}
\hline & Type " culard" $(\mathrm{C})$ & Type normal (N) & $\frac{\mathrm{C}-\mathrm{N}}{\mathrm{N}}$ p. 100 \\
\hline Poids vif (en kg) & 543 & 561 & $-3,2$ \\
\hline Poids de carcasse (en kg) & 361,4 & 333,6 & $+8,3$ \\
\hline $\begin{array}{l}\text { Poids total de muscle (kg) } \\
\text { de la demi-carcasse }\end{array}$ & 140,2 & 119,0 & $+17,8$ \\
\hline $\begin{array}{c}\text { Importance relative des muscles } \\
\text { (en pourcentage } \\
\text { de la musculature totale) }\end{array}$ & & & \\
\hline 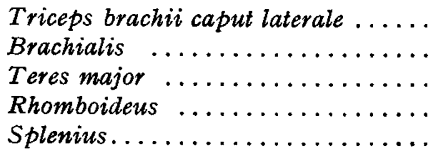 & $\begin{array}{l}0,72 \\
0,39 \\
0,42 \\
1,47 \\
0,68\end{array}$ & $\begin{array}{l}0,58 \\
0,45 \\
0,41 \\
1,30 \\
1,17\end{array}$ & $\begin{array}{r}+24,1 \\
-13,3 \\
+\quad 2,4 \\
+13,1 \\
-41,9\end{array}$ \\
\hline 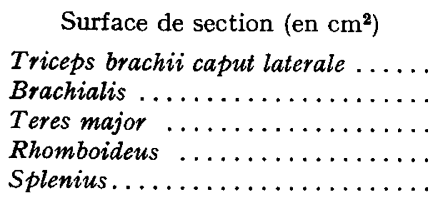 & $\begin{array}{l}96,62 \\
36,04 \\
41,93 \\
45,42 \\
58,03\end{array}$ & $\begin{array}{l}63,14 \\
34,54 \\
31,45 \\
41,07 \\
55,86\end{array}$ & $\begin{array}{l}+53,0 \\
+\quad 4,3 \\
+33,3 \\
+10,6 \\
+\quad 3,9\end{array}$ \\
\hline
\end{tabular}
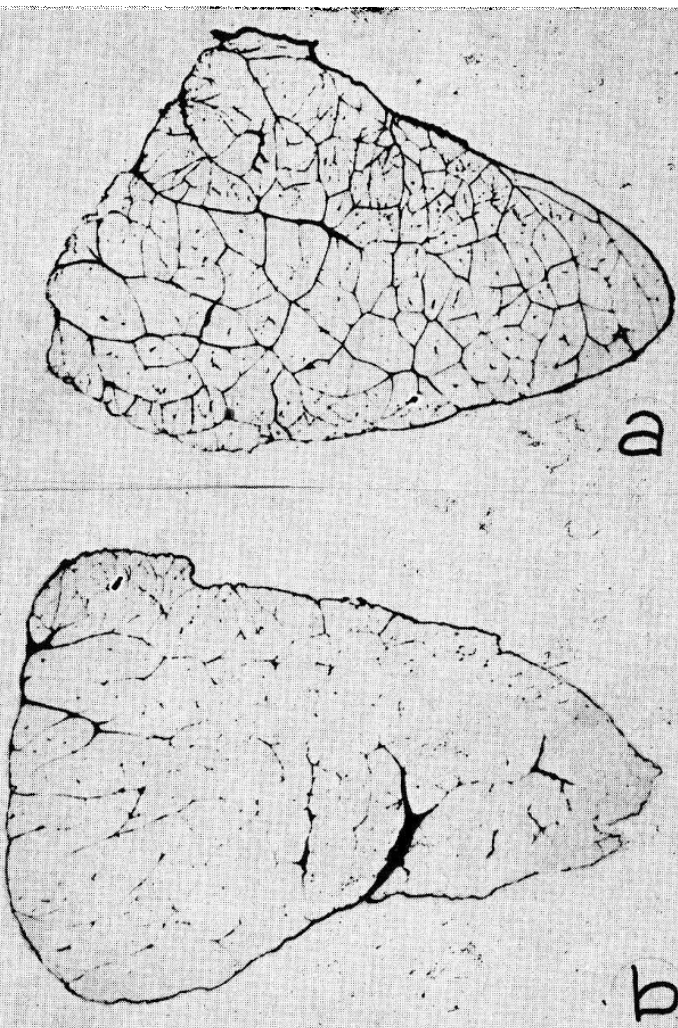

FIG. I. - Muscle Rhomboideus de bovin normal (a) et de bovin hypertrophié (b) $(\times \mathrm{I})$ 


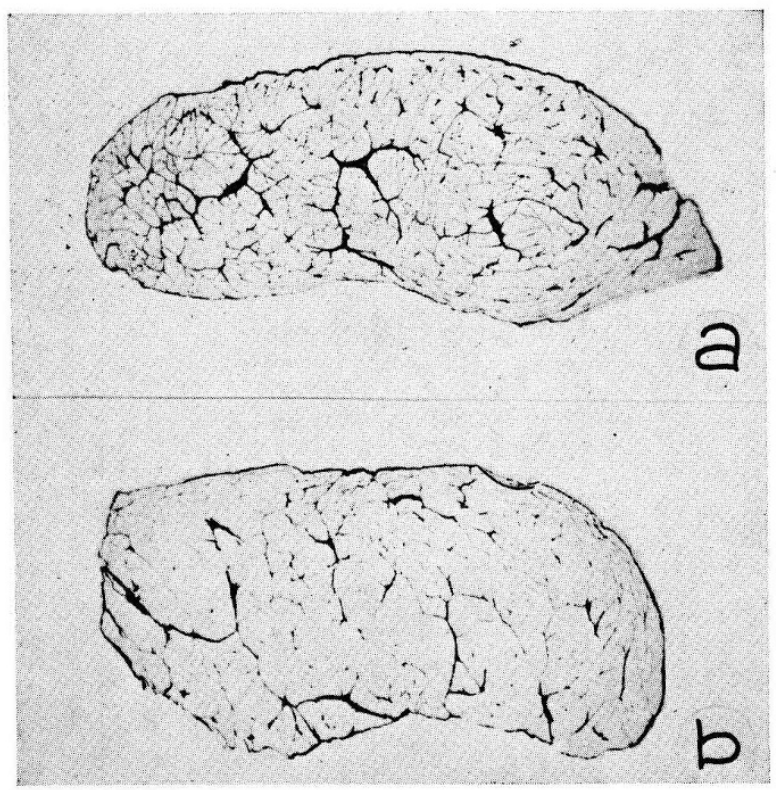

FIG. 2. - Muscle Brachialis de bovin normal (a) et de bovin hypertrophié (b)

$(\times I)$

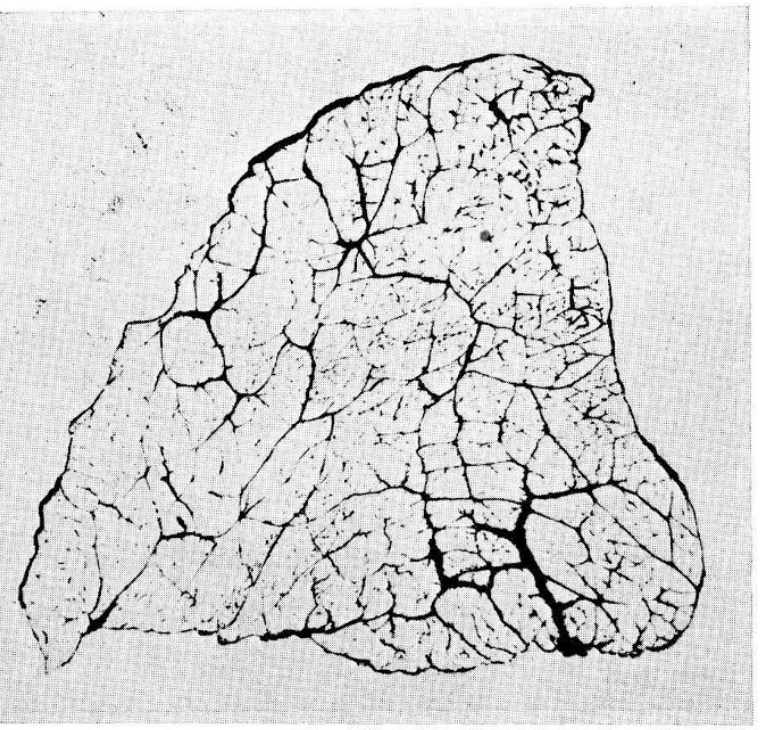

FIG. 3. - Muscle Triceps brachii, caput Iaterale de bovin normal $(\times I)$ 


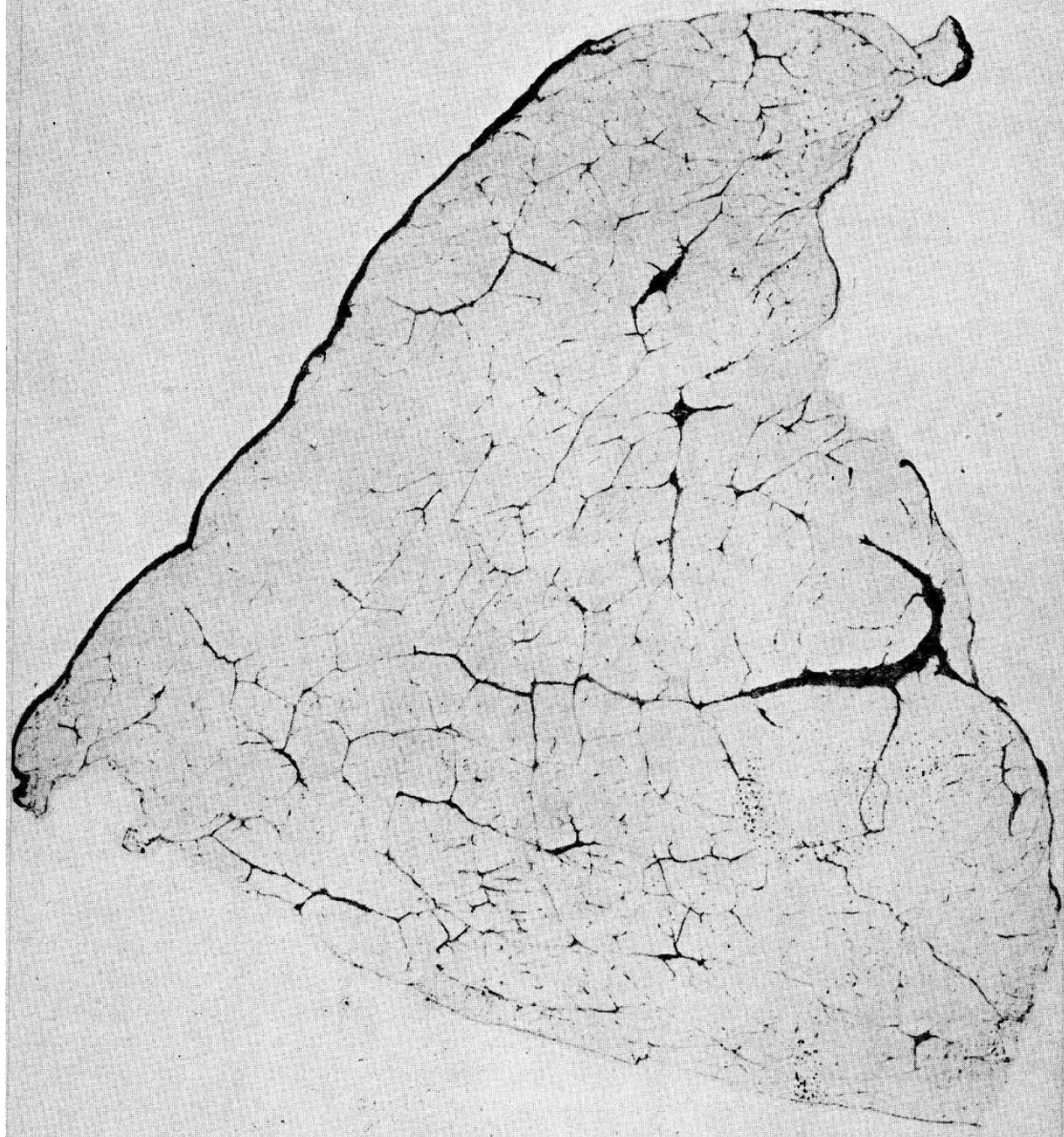

FIG. 4. - Muscle Triceps brachii, caput laterale de bovin hypertrophié $(\times \mathrm{I})$ 


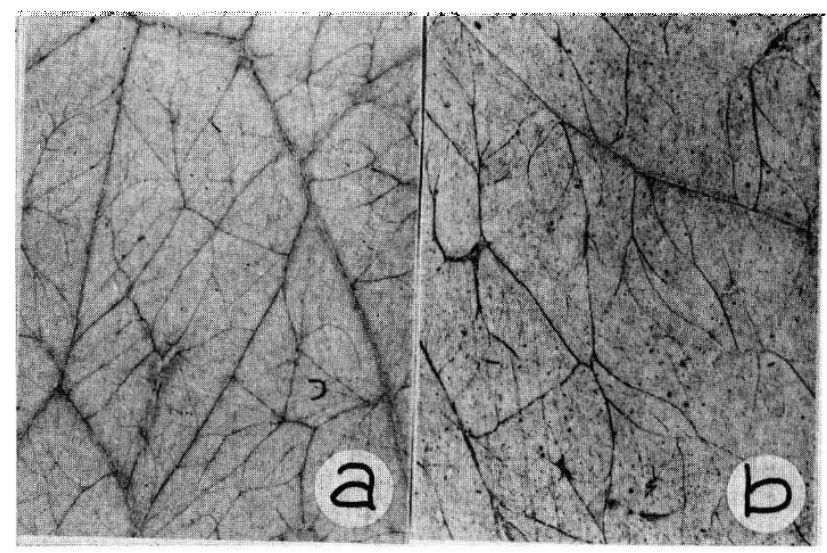

FIG. 5. - Coupe de muscle Splenius de bovin normal (a) et de bovin hypertrophié (b)

$(\times$ ro)

L'épaisseur de leurs parois est plus faible, au point de ne plus apparaître dans de nombreuses zones de la surface du muscle, alors que chez le sujet de type normal, dans les mêmes conditions d'observation des coupes, le réseau de tissu conjonctif est régulièrement visible. L'examen à plus fort grossissement révèle, en outre, que l'épaisseur des parois est relativement constante sur tout le pourtour d'un myoskhène donné, ce qui n'est généralement pas le cas dans le type normal.

D'autre part, aux sommets des unités, les " nœuds" de tissu conjonctif sont plus réduits dans le type hypertrophié.

L'accroissement de la taille des myoskhènes, perceptible sur les figures I à 4 , joint à la diminution de l'épaisseur moyenne de leurs parois contribue, chez l'animal hypertrophié, à accentuer la différence d'aspect entre les muscles des deux types et, peut-être, à sous-évaluer la quantité totale de collagène réellement présente dans les muscles.

Il apparaît donc que la trame générale de tissu conjonctif présente chez le bovin hypertrophié un aspect lâche très particulier, comparable à celui qui est observé dans le cas du muscle Semimembranosus chez les porcins de race Piétrain (Dumont et Schmitr, 1970). Si l'on admet que la dimension des myoskhènes est un facteur déterminant de l'aspect macroscopique de la texture de la viande, nos résultats sont en accord, sur ce point, avec les observations de Kidwell et al. (1952) pour lesquels la viande des bovins à musculature hypertrophiée est plus grossière.

Ce phénomène pourraît être un caractère très général, spécifique de l'hypertrophie musculaire héréditaire chaque fois que cette dernière se manifeste au moins sur le plan d'une hypertrophie morphologique, quelle que soit, par ailleurs, l'hypertrophie en masse du muscle considéré.

Un autre aspect important de l'organisation musculaire est l'abondance et la répartition du tissu conjonctif au sein de chaque myoskhène, où se définissent les faisceaux primaires, secondaires, tertiaires, etc... Dans un muscle de type normal (fig. 5) on perçoit des différences sensibles dans les épaisseurs des divers éléments du périmysium qui permettent de définir correctement la hiérarchie des faisceaux de fibres. Du pourtour des grandes unités, vers l'intérieur, se détachent des travées conjonctives qui dessinent un réseau dont les mailles deviennent progressivement de plus en plus fines, et dont les arborisations ultimes entourent les faisceaux primaires de fibres. Dans les muscles du bovin hypertrophié (fig. 5) ce phénomène ne se manifeste pas aussi nettement et de façon aussi progressive. A l'intérieur de chacun des myoskhènes, les parois du réseau conjonctif sont, d'une manière générale, très fines, et l'ensemble se présente de façon relativement uniforme. Il est souvent difficile d'y distinguer les différents ordres de faisceaux. Tous se passe comme 
si la hiérarchie de la distribution du tissu conjonctif était très fortement amoindrie, sinon complètement supprimée dans certaines zones des muscles. Ce phénomène semble être caractéristique de l'hypertrophie musculaire héréditaire de bovins car il apparaît de façon constante non seulement dans les muscles présentés dans ce travail mais dans un bon nombre d'autres muscles de "culards " de la même race examinés par ailleurs.

Cette répartition constitue une anomalie histologique notable qu'il est impossible que KIDWELl et al. (I952) aient pû ignorer si elle avait existé dans leur matériel expérimental, à moins que la dimension des échantillons histologiques qu'ils ont étudiés ait été trop faible.

Cette observation amène à souligner l'intérêt, pour le diagnostic et l'appréciation du caractère " culard " chez les bovins, de l'examen histologique, sur des coupes de grande surface, de la répartition du tissu conjonctif musculaire.

A notre connaissance, cet aspect particulier de la diffusion du conjonctif au sein des myoskhènes n'a pas été retrouvé jusqu'à présent dans d'autres espèces. Dans le cas du porc notamment, où certains types - tel le Piétrain - présentent des hypertrophies pondérales et morphologiques de divers muscles, nous n'avons pas observé ce phénomène.

Cette situation suggère que, sur le plan des caractères du périmysium, l'hypertrophie musculaire héréditaire a une manifestation différente selon les espèces.

Rę̧u pour publication en juin 1973.

\section{REMERCIEMENTS}

Nous remercions MM. Boccard et OuHAyoun pour les critiques et les suggestions qu'ils nous ont faites à la lecture du manuscrit.

\section{SUMMARY}

\section{EFFECTS OF THE HEREDITARY MUSCULAR HXPERTROPHY \\ ON THE CONNECTIVE TISSUE NETWORK}

The structure of muscular tissue in hypertrophied charollaise bullock of fifteen months old was shown to be very different from that of normal animal of same breed and age. In " culard" cattle it may exist an increase of the muscles cross section area independently of any weight hypertrophy. The same general trend was found in the different muscles whatever could be the relative degree of hypertrophy : the perimysium was less abundant in hypertrophied animal in which the connective tissue framework, defining the myoskhenes, was looser; in each of these muscular unit there was a rather uniform distribution of connective tissue so that many often it was very difficult to distinguish types of muscle fiber bundles other than primary ones.

The relationships between muscular hypertrophy and histological structure are discussed ant it is suggested that if the characters of perimysium (amount, distribution, depth at different levels) are influenced by muscular hypertrophy, the phenomenon is not quite the same in different species.

\section{RÉFÉRENCES BIBLIOGRAPHIQUES}

Boccard R., r964. Relations entre l'hypertrophie musculaire héréditaire des bovins culards et le métabolisme du collagène. Ann. Zootech., 13, 389-39r.

Boccard R., Dumont B. L., Schmits O., I 969 . Caractéristiques différentielles du tissu conjonctif des bovins normaux et " culards ". Ann. génét. Sél. anim., 1, I78. 
Dumont B. L., Schmits O., I97o. Anatomie microscopique comparée du tissu musculaire squelettique de porcs Large White et Piétrain. Ann. Génét. Sél. anim., 2, 381-39r.

Dúmont B. L., Boccard R., I972. Conséquences de l'hypertrophie musculaire héréditaire des bovins sur la musculature. II. Importance relative des différents muscles. Ann. Génét. Sél. anim., I972, 4, I3O-I3I.

Kidwell J.F., Vernon E. H., Crown R. M., Singletary C.B., I952. Muscular hypertrophy in cattle. J. Hered., 43, 62-68.

Lawrie R. A., Pomeroy R. W., Williams D. R., ig64. Studies in the muscles of meat animals. IV. Comparative composition of muscles from "doppelender " and normal sibling heifers. J.agric. Sci., 62, 89-92.

Ouhayoun J., Beaumont A., r968. Étude du caractère culard. III. Anatomie microscopique comparée du tissu musculaire de mâles charolais normaux et culards. Ann. Zootech., 17, 217-223.

Schmitт O., Dumont B. L., 1969. Méthodes d'analyse de la structure musculaire. Ann. Biol. anim. Bioch. Biophys., 9, 123-1 34 . 